\title{
Normalização EMG: considerações da literatura para avaliação da função muscular
}

\section{EMG normalization: considerations of the literature for muscular function evaluation}

\author{
Rubens Alexandre da Silva Jr. \\ Pós-Doutorado - Elisabeth Bruyere Research Institute/University of Ottawa. Doutor em Reabilitação e Ciências Biomédicas - \\ Université de Montréal. Pesquisador - Centro de Pesquisa em Ciências da Saúde. Coordenador Laboratório de Avaliação Funcional \\ e Performance Motora Humana (LAFUP) - Universidade Norte do Paraná - Unopar. Londrina, PR. Professor Titular - Programa de \\ Mestrado e Doutorado em Ciências da Reabilitação - Universidade Estadual de Londrina/ Universidade Norte do Paraná - UEL/ \\ Unopar. Londrina, PR - Brasil.
}

Endereço para correspondência

Rubens Alexandre da Silva Jr.

Av. Paris, 675, Jardim Piza

86041-140 - Caixa Postal 401 - Londrina - PR [Brasil]

rubens@unopar.br

\section{Resumo}

Introdução: Diferentes fatores intrínsecos e extrínsecos influenciam a medida de atividade elétrica muscular por meio da eletromiografia (EMG). Para minimizar este efeito, a normalização da EMG é recomendada. Entretanto, pouco se sabe sobre as vantagens e desvantagens de cada método de normalização para uso em diferentes pacientes (sintomáticos e assintomáticos). Objetivos: Revisar e discutir os principais métodos de normalização do sinal da EMG para avaliação da função motora em diferentes sujeitos. Métodos: Incluíram-se artigos publicados, desde 1984, constantes na base de dados Medline. Dezessete estudos, sendo dois com pacientes sintomáticos, e dois, de revisão, foram selecionados para revisão crítica. Conclusão: A escolha de uma técnica de normalização da EMG é com base na variabilidade entre e intra-indivíduos. Um método recomendado para reduzir a variabilidade biológica é aquele com base numa contração voluntária submáxima como referência no processo de normalização. Entretanto, este não considera a capacidade máxima muscular do indivíduo para execução de uma atividade motora.

Descritores: Avaliação; Biomecânica; Eletromiografia; Músculos.

\begin{abstract}
Introduction: Different intrinsic and extrinsic factors may affect the electrical muscular activity using the electromyography (EMG). To minimize this effect, the normalization of the EMG is suggested. However, it is not clear the advantages and disadvantages of each EMG normalization method when applied in different patients (symptomatic and asymptomatic). Objectives: To review and discuss the main methods of normalization of the EMG signal for evaluation of the motor function in different individuals. Methods: This revision included scientific papers since 1984, available on Medline databases. Seventeen articles, being two with symptomatic patients and two of revision, were selected for the critical review. Conclusion: The choice of a normalization technical is based on intra-and inter-individual variability. One method more suitable to reduce the biological variability is of a sub-maximal voluntary contraction, used as reference on EMG normalization. However, using this method there is a lack of information corresponding to the level of muscle activation required for the task in relation to the maximal capacity of individual.
\end{abstract}

Key words: Biomechanics; Electromyography; Evaluation; Muscles. 


\section{Introdução}

A eletromiografia (EMG) é o registro da atividade elétrica dos músculos. Este registro é feito por meio dos eletrodos de superfície (posicionados sobre a pele) ou de modo intramuscular. O registro do sinal eletromiográfico constitui-se de um somatório de várias séries de potencial de ação das unidades motoras (UM) dentro das fibras musculares durante a contração muscular. É possível analisar o sinal da EMG, tanto no domínio temporal como em frequência. No que se refere aos parâmetros da EMG no domínio do tempo, uma medida preconizada na literatura é a amplitude do sinal da EMG, chamada de Root Mean Square (RMS)2. Outros parâmetros, tais como amplitude da EMG integrada e envelope linear, também são utilizados para avaliar a ativação muscular ${ }^{2}$. Em geral, a medida que a força aumenta, durante uma contração voluntária isométrica máxima, a amplitude do sinal da EMG também aumenta, caracterizando então a relação EMG-força ${ }^{1,2}$.

A força muscular varia em relação a quantidade de UMs ativas. De fato, existem dois mecanismos para aumentar a força produzida por um músculo durante uma contração muscular voluntária, a saber: (1) aumentar o número de UMs ativas (recrutamento) e (2) aumentar a frequência de disparo das UMs já ativas no músculo. O primeiro conceito segue a ordem específica de recrutamento dos motoneurônios, denominado size principle ${ }^{3}$. Conforme este princípio, o recrutamento de motoneurônios é ordenado estritamente em razão do tamanho: os menores motoneurônios, os quais os axônios produzem menores potenciais de ação e produzem pouca tensão muscular (slow twitch, fatigue resistant), são recrutados em primeiro lugar; em seguida, os maiores motoneurônios associados às UMs que produzem maior tensão muscular, mas com pouca resistência a fadiga (fast twitch, fast-fatiguing $)^{3}$. A medida EMG em RMS é geralmente a medida mais utilizada para quantificar fisiologicamente estes mecanismos de força muscular ${ }^{1}$.
Entretanto, diferentes fatores intrínsecos e extrínsecos podem afetar essa medida e, consequentemente, influenciar a relação EMG-força durante a contração muscular voluntária. Estes fatores se caracterizam por: temperatura da pele, quantidade de tecido adiposo, configuração dos eletrodos, localização dos eletrodos em relação às fibras musculares e distância intereletrodos ${ }^{1}$. A influência desses fatores nas medidas da EMG prejudica na comparação dos resultados entre diferentes grupos de indivíduos (com disfunções neuromusculares, adultos saudáveis, idosos, atletas) e/ou entre diferentes grupos musculares ${ }^{1,2,4}$. Para minimizar esta situação, a normalização da amplitude do sinal da EMG é recomendada. Este procedimento é realizado após o processamento do sinal e pode ser dependente das condições experimentais, tais como um protocolo de contração muscular máxima e/ ou submáxima. Recentemente, uma revisão da literatura ${ }^{4}$ apresentou os diferentes métodos de normalização empregados para determinar a magnitude de ativação muscular, mas com ênfase em indivíduos saudáveis. A discussão sobre o uso dos principais métodos de normalização para indivíduos com disfunção é ainda pouco abordada na literatura sobre o assunto. O objetivo deste estudo é proporcionar aos leitores o conhecimento sobre o que a literatura traz sobre a normalização da EMG em pacientes com disfunções neuromusculares, revisar conceitos e discutir as vantagens e desvantagens de cada qual para uso em diferentes populações de pacientes, assim como na abordagem de indivíduos saudáveis.

\section{Métodos}

Trata-se de um estudo de revisão crítica e integrativa da literatura sobre a normalização do sinal da EMG para avaliação da função muscular. Foram consultados periódicos indexados na base de dados PubMed (US National Library of Medicine National Institutes of Health: http:// www.ncbi.nlm.nih.gov/pubmed). PubMed com- 
preende mais de 22 milhões de citações para literatura biomédica com base Medline, revistas para ciências da vida e livros online. Esta base é considerada hoje uma das mais importantes do mundo para ciências da saúde. Os descritores utilizados para este trabalho foram termos em inglês apenas, tais como Electromyography, EMG Normalization, Isometric MVC, Muscular Utilization Ratio, Inter-Individual variability. Para descrever a revisão crítica, 17 artigos científi$\cos ^{4-20}$, sendo dois contendo pacientes sintomá$\operatorname{ticos}^{8,19}$, e dois, de revisão da literatura ${ }^{4,10}$, foram selecionados, conforme mostra a Tabela 1, para explanar o principal tema abordado: "normalização EMG". Os demais artigos referidos na bibliografia são complementares para redação crítica do assunto e entendimento da área. Os estudos específicos sobre a normalização EMG, citados neste trabalho, foram publicados entre os anos de 1984 e 20104-20.

\section{Resultados}

Os principais métodos de normalização citados na Tabela 1, correspondem ao método de

Tabela 1: Principais estudos sobre a normalização do sinal da eletromiografia desde 1984

\begin{tabular}{|c|c|c|c|}
\hline Autor/Ano & Objetivo & Amostra & Conclusão \\
\hline $\begin{array}{l}\text { Yang JF e } \\
\text { Winter DA } \\
(1984)^{5}\end{array}$ & $\begin{array}{l}\text { Analisar os efeitos de quatro } \\
\text { métodos de normalização da } \\
\text { amplitude da EMG na variabilidade } \\
\text { inter-indivíduos durante a marcha. }\end{array}$ & $\begin{array}{l}\mathrm{n}=11 \text { indivíduos } \\
\text { saudáveis. }\end{array}$ & $\begin{array}{l}\text { A redução da variabilidade inter-indivíduos } \\
\text { é possível pela normalização da EMG, } \\
\text { utilizando tanto o pico quanto a média do } \\
\text { sinal EMG da própria tarefa de marcha. }\end{array}$ \\
\hline $\begin{array}{l}\text { Mirka GA } \\
(1991)^{6}\end{array}$ & $\begin{array}{l}\text { Quantificar os erros associados com } \\
\text { normalização da EMG, quando normalizado } \\
\text { em uma posição articular arbitrária durante } \\
\text { os esforços do tronco em extensão. }\end{array}$ & $\begin{array}{l}\mathrm{n}=4 \text { indivíduos } \\
\text { saudáveis. }\end{array}$ & $\begin{array}{l}\text { Erros de } 75 \% \text { do valor esperado pode ser } \\
\text { alcançados durante tarefas dinâmicas do } \\
\text { tronco, quando normalizando a EMG com } \\
\text { apenas um ponto angular de referência. }\end{array}$ \\
\hline $\begin{array}{l}\text { Allison, GT, } \\
\text { Marshall RN, } \\
\text { Singer KP } \\
(1993)^{7}\end{array}$ & $\begin{array}{l}\text { Determinar o efeito de } 11 \text { métodos } \\
\text { de normalização da EMG, usando o } \\
\text { coeficiente de variação (CV), durante } \\
\text { ciclos de alongamento-encurtamento } \\
\text { (atividade concêntrica e excêntrica), } \\
\text { do músculo bíceps braquial, em } \\
\text { velocidades e cargas variáveis. }\end{array}$ & $\begin{array}{l}\mathrm{n}=8 \text { indivíduos } \\
\text { saudáveis. }\end{array}$ & $\begin{array}{l}\text { A normalização da EMG pela contração } \\
\text { isométrica voluntária máxima (RMS } \\
\text { aumenta o coeficiente de variação durante } \\
\text { os ciclos de alongamento-encurtamento. } 0 \\
\text { coeficiente de variação é reduzido quando } \\
\text { o sinal é normalizado por um contração } \\
\text { voluntária submáxima ou pela média do } \\
\text { sinal EMG, durante a própria tarefa motora. }\end{array}$ \\
\hline $\begin{array}{l}\text { Knutson LM, } \\
\text { Soderberg GL, } \\
\text { Ballantyne } \\
\text { BT, Clarke WR } \\
(1994)^{8}\end{array}$ & $\begin{array}{l}\text { Determinar qual dos três métodos de } \\
\text { normalização da EMG fornece dados } \\
\text { mais reprodutíveis. Foram analisados a } \\
\text { contração isométrica voluntária máxima } \\
\text { (CIVM), o pico dinâmico da EMG e a } \\
\text { média da EMG dinâmico do músculo } \\
\text { gastrocnêmico, durante a marcha. }\end{array}$ & $\begin{array}{l}\mathrm{n}=20 \text { indivíduos } \\
\text { saudáveis; } \\
\mathrm{n}=20 \text { indivíduos } \\
\text { com lesão do } \\
\text { ligamento cru- } \\
\text { zado anterior. }\end{array}$ & $\begin{array}{l}\text { Conforme o índice de variação e o } \\
\text { coeficiente de correlação intraclasse } \\
\text { (CCI), a reprodutibilidade foi melhor } \\
\text { apontada quando a normalização era } \\
\text { realizada pela CIVM. Já o coeficiente } \\
\text { de variação suportou o uso do pico } \\
\text { dinâmico da própria atividade motora } \\
\text { para melhor precisão dos dados. }\end{array}$ \\
\hline
\end{tabular}

Comparar três procedimentos de normalização da amplitude da EMG no músculo trapézio superior. Os métodos foram : (a)

Attebrant M, normalizar por meio de uma CIVM em Mathiassen rampa; (b) normalizar por meio de uma SE, Winkel J carga relativa constante proveniente da $(1995)^{9} \quad$ CIVM e (c) normalizar mediante contração isométrica voluntária submáxima como referência (e.g. percentage of a reference voluntary electrical activation: \%RVE). $\mathrm{n}=15$ indivíduos saudáveis.
Menor variabilidade interindivíduos foi encontrada por meio do método \%RVE, o qual é proveniente de uma contração voluntária submáxima. Os demais métodos aumentam esta variabilidade $\mathrm{e}$ influenciam os resultados de medida. 
Cont. Tabela l: Principais estudos sobre a normalização do sinal da eletromiografia desde 1984

\begin{tabular}{lll}
\hline Autor/Ano & Objetivo & Amostra \\
\hline & & Foram apontados \\
& & 77 estudos entre \\
& & os anos de 1978 a \\
& Realizar uma revisão de diferentes & 1994. Do total, 45 \\
& procedimentos de normalização & se caracterizam \\
& da EMG para o músculo trapézio & da EMG de \\
Mathiassen & superior. Os principais problemas & origem neurofisio- \\
SE, Winkel & abordados na revisão foram: variável & lógica (com base na \\
J, Hagg GM & de medida, posição dos eletrodos, & ativação muscular \\
$(995)^{10}$ & postura do indivíduo durante & por meio das \\
& o teste, movimento, carga, duração & unidades motoras). \\
& das contrações musculares, & Os demais focam \\
& processamento dos sinais & na normalização da \\
& da EMG e a confiabilidade & força mecânica pro- \\
& teste-reteste. & duzida pelo torque \\
& & muscular, durante \\
& & uma contração \\
& & voluntária. \\
\hline
\end{tabular}

\begin{tabular}{lll}
\hline Harms- & Analisar se os métodos de normalização & \\
Ringdahl & influencia na interpretação da carga & \\
K, Ekholm & músculo do trapézio superior, durante & $\mathrm{n}=9$ pilotos de \\
J, Schuldt & uma tarefa ergonômica. Dois valores de & jato saudáveis \\
K, Linder J, & referência para normalização da EMG & entre 30 a \\
Ericson MO & foram testadas, a saber (1) com base na & 48 anos \\
$(1996)^{11}$ & CIVM e (2) com base numa carga muscular & \\
& padronizada tal como o Dumbbell test. & \\
\hline
\end{tabular}

Comparar diferentes métodos de Kellis E e normalização da atividade eletromiográfica BaltzopoulosV (EMG) dos músculos antagonistas do $(1996)^{12} \quad$ joelho, durante movimentos isocinéticos em modo concêntrico e excêntrico.

$\mathrm{n}=20$ mulheres

jovens saudáveis.
Conclusão

Existe falta de consenso sobre as terminologias empregadas para normalização da EMG. É proposta uma terminologia padrão relacionada à atividade elétrica muscular em amplitude do sinal EMG, mas que refere-se as atividades ergonômicas para avaliação do músculo trapézio superior, considerando fatores como postura, carga e duração da contração muscular.
A normalização pela CIVM é mas adequada para se estabelecer as sobrecargas musculares de trabalho com base as capacidades físicas entre os indivíduos, quando comparado à normalização por meio de um teste padronizado de carga submáxima, no qual poderia apenas inferir alguma mudança no grau de sobrecarga.

Os resultados demonstraram o efeito dos métodos de normalização, sendo o método dinâmico de normalização da EMG o mais apropriado, pois este considera os efeitos da ação muscular (comprimento, velocidade angular).

Ambos os métodos de processamento e análise da EMG apresentaram excelente confiabilidade (coeficientes de correlação intraclasse $>0,86$ ).
Zacaria D, Kramer JF, Harburn KL $(1996)^{13}$ Avaliar a confiabilidade teste-reteste do EMG bruto e normalizado em \% por meio da CIVM, durantes esforços de extensão do joelho (músculos vasto medial e vasto lateral).

Avaliar e comparar quatro métodos

Burden A e Bartlett R $(1999)^{14}$ de normalização da amplitude da eletromiografia (EMG) do músculo bíceps braquial. $\mathrm{n}=20$ homens

jovens

saudáveis.
Concluiu-se que o método de contração voluntária máxima (CVM), no modo isométrico ou isocinético, é o mais adequado para representar a atividade muscular do indivíduo com base na sua capacidade máxima para realização da tarefa motora.

0 s resultados mostraram semelhanças entre o verdadeiro valor de torque máximo em relação ao valor predito na equação com base nas características antropométricas de indivíduos saudáveis. É sugerido que este método (torque máximo predito + EMG predito) poderia ser utilizado como referência no procedimento de normalização da EMG.
Marras WS e Davis KG $(2001)^{15}$
Desenvolver uma técnica de normalização capaz de predizer a força muscular máxima. Equações de regressão com base em medidas antropométricas forma utilizadas para estimar o esforço máximo do indivíduo. $\mathrm{n}=20$ indivíduos saudáveis. 
Cont. Tabela l: Principais estudos sobre a normalização do sinal da eletromiografia desde 1984

\begin{tabular}{|c|c|c|c|}
\hline Autor/Ano & Objetivo & Amostra & Conclusão \\
\hline $\begin{array}{l}\text { Marras WS, } \\
\text { Davis KG, } \\
\text { Maronitis AB } \\
(2001)^{16}\end{array}$ & $\begin{array}{l}\text { Verificar se o método de estimação da } \\
\text { contração voluntária máxima é capaz de } \\
\text { caracterizar as capacidades musculares } \\
\text { dos indivíduos. Determinar se o método } \\
\text { poderia ser empregado em pacientes } \\
\text { com dor lombar crônica. Comparou-se a } \\
\text { tradicional normalização EMG por meio } \\
\text { da CIVM; e o modelo EMG estimado, } \\
\text { por esforços submáximos, durante } \\
\text { tarefas de levantamento de cargas. }\end{array}$ & $\begin{array}{l}\mathrm{n}=20 \text { indivíduos } \\
\text { saudáveis ( } 10 \\
\text { homens e } 10 \\
\text { mulheres). }\end{array}$ & $\begin{array}{l}\text { Com base nestes resultados, } \\
\text { o método de normalização proposta } \\
\text { (modelo EMG-assistido) pode ser } \\
\text { viável para a normalização da EMG, no } \\
\text { intuito de avaliar indivíduos incapazes } \\
\text { de produzir o esforço máximo. }\end{array}$ \\
\hline $\begin{array}{l}\text { Benoit DL, } \\
\text { Lamontagne } \\
\text { M, Cerulli G, } \\
\text { Liti A }(2003)^{17}\end{array}$ & $\begin{array}{l}\text { Analisar três métodos de normalização } \\
\text { da EMG para detectar alterações } \\
\text { neuromusculares em pacientes com } \\
\text { lesão do ligamento cruzado anterior, } \\
\text { durante a marcha na esteira. }\end{array}$ & $\begin{array}{l}\mathrm{n}=12 \text { homens } \\
\text { e } 4 \text { mulheres } \\
\text { saudáveis. }\end{array}$ & $\begin{array}{l}0 \text { método da CIVM é o mais sensível } \\
\text { para detectar as diferenças entre os } \\
\text { grupos para interpretação clínica das } \\
\text { alterações neuromusculares com base } \\
\text { na capacidade máxima do indivíduo. }\end{array}$ \\
\hline $\begin{array}{l}\text { Burden AM, } \\
\text { Trew M, } \\
\text { Baltzopoulos } \\
\text { V }(2003)^{18}\end{array}$ & $\begin{array}{l}\text { Comparar quatro diferentes } \\
\text { métodos de normalização da EMG, } \\
\text { durante a marcha. }\end{array}$ & $\begin{array}{l}\mathrm{n}=10 \text { homens } \\
\text { e } 10 \text { mulheres } \\
\text { saudáveis. }\end{array}$ & $\begin{array}{l}\text { O método de CVM, no modo isocinético, } \\
\text { não reduz a variabilidade intra- ou } \\
\text { interindivíduos em comparação com os } \\
\text { demais métodos. O CIVM, ou seja, durante } \\
\text { uma contração isométrica, fornece } \\
\text { uma medida mais representativa da } \\
\text { ativação muscular, durante a marcha. }\end{array}$ \\
\hline $\begin{array}{l}\text { Ekstrom RA, } \\
\text { Soderberg GL, } \\
\text { Donatelli RA } \\
(2005)^{19}\end{array}$ & $\begin{array}{l}\text { Quantificar a atividade da EMG dos } \\
\text { músculos serrátil anterior e trapézio } \\
\text { (superior, medial e inferior), durante } \\
\text { nove testes musculares manuais } \\
\text { realizados com esforço máximo. }\end{array}$ & $\begin{array}{l}\mathrm{n}=30 \text { indivíduos } \\
\text { saudáveis. }\end{array}$ & $\begin{array}{l}\text { Este estudo preconiza algumas posturas } \\
\text { específicas para elevar o recrutamento } \\
\text { dos músculos avaliados para uso da CIVM } \\
\text { no processo de normalização da EMG. }\end{array}$ \\
\hline $\begin{array}{l}\text { Milot MH, } \\
\text { Nadeau S, } \\
\text { Gravel D } \\
(2007)^{20}\end{array}$ & $\begin{array}{l}\text { Avaliar a taxa de utilização muscular (TUM) } \\
\text { de flexores plantares, flexores do quadril } \\
\text { e os músculos extensores do quadril, } \\
\text { durante sua ação concêntrica, na marcha } \\
\text { com velocidades autosselecionadas. }\end{array}$ & $\begin{array}{l}\mathrm{n}=17 \text { participan- } \\
\text { tes hemiparéti- } \\
\text { cos crônicos. }\end{array}$ & $\begin{array}{l}\text { Pôde-se concluir que os grupos musculares } \\
\text { paréticos mais fracos mostraram maior } \\
\text { porcentagem de esforço, durante a } \\
\text { marcha, quando normalizados pela CIVM. }\end{array}$ \\
\hline $\begin{array}{l}\text { Burden A } \\
(2010)^{4}\end{array}$ & $\begin{array}{l}\text { Analisar por meio de uma revisão oito } \\
\text { métodos de normalização da EMG } \\
\text { com base na confiabilidade do dados } \\
\text { e na variabilidade interindivíduos. }\end{array}$ & $\begin{array}{l}\text { Foram } \\
\text { encontrados } 26 \\
\text { estudos, mas } \\
\text { somente } 16 \\
\text { foram discutidos } \\
\text { com base na } \\
\text { escolha do valor } \\
\text { de referência. }\end{array}$ & $\begin{array}{l}\text { A normalização da EMG por meio da CIVM } \\
\text { é confiável, quando por uma contração } \\
\text { voluntária submáxima, e não aparece ser } \\
\text { afetada pelo tipo de contração muscular } \\
\text { da tarefa e ângulo articular, quando estes } \\
\text { são bem controlados. Alternativamente, } \\
\text { o método dinâmico também pode ser } \\
\text { utilizado, mas não se mostra superior ao } \\
\text { CIVM mesmo para tarefas dinâmicas. }\end{array}$ \\
\hline
\end{tabular}

normalização por meio: (a) da ativação elétrica voluntária máxima ${ }^{4,9-14,16-20}$; (b) da ativação elétrica voluntária submáximat,7,9-11,16,18; (c) de contrações dinâmicas ${ }^{4-6,8,10,12,17,18,20}$ e (d) em alguns casos, por intermédio da estimação da força muscular máxima proveniente de medidas antropométricas ${ }^{15,16}$.

Conforme os estudos apontados, quatro principais métodos de normalização são propostos. O primeiro é o método de normalização da
EMG por meio da porcentagem de ativação máxima que é definido pelos esforços de Contração Voluntária Máxima (CVM), de maneira isométrica (CIVM) ou dinâmica (CVM dinâmica), dependendo da tarefa motora ${ }^{4,10}$. O segundo método de normalização da EMG é por ativação elétrica submáxima ${ }^{4,10}$, que é utilizado durante esforços de contração submáxima somente, conforme protocolo padronizado de carga absolu- 
ta inferior ao máximo ou proveniente da \% relativa do máximo (ex: $20 \%, 30 \%, 40 \%$ ou $50 \%$ ). Em terceiro, a literatura propõe também outros métodos de normalização específicos para contrações dinâmicas, especialmente para marcha ${ }^{5}$. Esses foram implantados durante as análises do padrão de marcha para obter-se um padrão normal de atividade dos músculos dos membros inferiores e possibilitar, assim, a comparação entre indivíduos diferentes. Finalmente como quarto procedimento, um grupo de pesquisadores tentou predizer os esforços máximos dos músculos do tronco ao invés de usar uma CVM como referência no procedimento de normalização ${ }^{15,16}$. A predição dos esforços máximos foi realizada a partir de medidas antropométricas dos sujeitos.

Todos esses estudos apresentavam algo em comum: determinar um método capaz de preservar as variâncias biológicas e, ao mesmo tempo, minimizar os efeitos da variabilidade intra- e interindivíduos. Cada método de normalização da EMG foi proposto para uma atividade motora padrão a fim de discriminar a ativação muscular de um grupo muscular alvo em diferentes sujeitos (Figura 1). Entre os trabalhos analisados, somente em dois avaliaram-se indivíduos sintomáticos ${ }^{8,16}$, sendo pacientes com lesão de joelho (ortopédico) ou com acidente vascular encefálico (hemiparéticos). Em ambos os estudos, o método preconizado foi a CIVM para discriminar os grupos de indivíduos quanto às alterações neuromusculares. As demais pesquisas envolviam pessoas saudáveis jovens (em média 25 anos). Duas tratavam de revisão dos achados, sendo a mais atual de Burden ${ }^{4}$. Este autor enfatizou, para sujeitos saudáveis, a normalização da EMG por meio da CIVM como método confiável tanto para tarefas isométricas quanto dinâmicas, como a marcha.

Todos os estudos não identificaram especificamente as vantagens e desvantagens de cada técnica, quando portadores de alteração neuromusculoesquelética são avaliados. Este tópico será discutido, na sequência, juntamente com os conceitos e definições de cada técnica de normalização para uma determinada tarefa motora.

\section{Discussão}

Na maioria dos estudos, o método mais utilizado como referência para o procedimento de normalização da amplitude do sinal da EMG é por meio da CIVM ${ }^{9-11}$. Este método é capaz de quantificar de maneira precisa o esforço relativo dos grupos musculares e permiti a comparação entre os indivíduos com e sem disfunção neuromuscular. É também definido por alguns autores ${ }^{20}$ como taxa de utilização muscular (em \%), Muscular Utilization Ratio, caracterizado pela relação entre a demanda mecânica imposta durante a atividade motora e a capacidade máxima do grupo muscular efetuar a atividade. Esta relação é multiplicada por cem para obter-se uma taxa em porcentagem (\%), com base na capacidade máxima, para desenvolver uma tarefa motora específica.

É importante mencionar que este método pode ser influenciado, em indivíduos sintomáticos, em razão de um problema neuromusculoesquelético ${ }^{21}$. A amplitude RMS máxima calculada, por exemplo, representa o denominador na equação e corresponde à ativação mais alta que pode produzir o músculo, durante uma CVM. Todavia, se o sujeito apresentar muita dor ou medo de realizar o movimento no decorrer do teste, este denominador não representará a real informação biológica. A literatura relata que pacientes com lombalgia crônica, por exemplo, têm medo de desencadear a dor ou de se lesionar durante os esforços de extensão do tronco ${ }^{21}$. Neste caso, o registro da atividade do músculo leva em consideração a capacidade do paciente em tolerar sua dor e mesmo sua motivação em fornecer um esforço máximo, o que se traduz por um falso denominador. Consequentemente, esse mecanismo de defesa do indivíduo com dor pode subestimar o esforço relativo do músculo avaliado. Uma alternativa para sanar este problema é utilizar uma tarefa funcional padronizada ${ }^{11}$, ou uma contração submáxima ${ }^{9}$, ou, até mesmo, estimar os esforço relativos por medidas antropométicas ${ }^{16}$. 
Outro fator importante a controlar na utilização do método da CIVM é o comprimento dos músculos. O comprimento muscular (ou o ângulo articular) deve ser bem controlado, especialmente em tarefas dinâmicas, em que a posição relativa entre o eletrodo e o músculo se modifica em razão do movimento da pele; assim como a quantidade do sinal da EMG que pode variar em função do número de UMs ativas sob o eletrodo ${ }^{1}$. Para estudar a marcha, alguns autores $^{5,22}$ adotaram o método de calibração de referência por meio de uma CVM no modo isométrico. Esta técnica é, entretanto, questionada se o ângulo de referência durante a CVM não for correspondente aos ângulos avaliados durante a tarefa dinâmica. Mirka ${ }^{26}$ apresentou grande margem de erros dentro da normalização da EMG para os músculos paravetebrais, quando o ângulo de referência era estabelecido de forma arbitrária e sem correspondência com o ângulo de esforços dinâmicos de flexão e extensão do tronco. É importante ressaltar os recursos tecnológicos que podem ser utilizados em paralelo ao sistema de eletromiografia durante a coleta de dados em benefício do controle angular, tais como eletrogoniômetros e acelerômetros, que serviriam para minimizar esses efeitos. Em trabalhos recentes ${ }^{23,24}$, desenvolveram-se muito bem este paralelismo instrumental em situação dinâmica e mostraram-se resultados interessantes na comparação entre diferentes grupos musculares, isolando cada qual e respeitando seus comprimentos musculares, na determinação de melhores exercícios para fortalecer os músculos da região lombar. Estes autores utilizaram o método de CIVM para expressar a \% de ativação dos músculos durante exercícios dinâmicos do tronco.

Em relação à contração submáxima como referência na normalização, alguns estudos mostraram melhor confiabilidade da medida com o uso deste método referente à CVM, particularmente na avaliação de pacientes com disfunções musculoesqueléticas ${ }^{25}$. Entretanto, diferenças podem ser observadas quando a normalização é realizada com uma carga submáxi- ma relativa à CVM, ou quando a normalização é realizada com base em uma carga submáxima absoluta. De fato, se a carga de referência é relativa da CVM, é possível que a estimação do esforço seja também problemática visto que pacientes com disfunções musculoesqueléticas não produziriam um verdadeiro esforço devido a seus sintomas de dor e/ou medo de realizar o movimento. Consequentemente, o produto final é subestimado. Uma alternativa para controlar essa problemática é utilizar uma contração de referencia de carga absoluta ou relativa a um parâmetro antropométrico ao invés de uma carga relativa da CVM. Um estudo ${ }^{26}$ mostrou a eficácia deste procedimento de normalização em pacientes com sintomas de lombalgia e indivíduos assintomáticos. Os autores ${ }^{26}$ utilizaram um teste de referência (denominador) com base em uma carga absoluta referente a $40 \%$ do peso corporal do indivíduo. Outra forma, é usar um valor de referência do próprio exercício funcional submáximo, ou seja, calculando-se o peak do valor RMS durante o esforço submáximo ${ }^{4}$. Ambas as técnicas de forma submáxima contribuem a reduzir a variabilidade entre os pacientes em relação aos valores brutos do sinal da EMG, mas não revela toda a variância biológica da atividade muscular em relação à capacidade máxima.

Além de CVM e de contração submáxima, a literatura propõe outros métodos de normalização específicos para contrações dinâmicas. Novamente, estas técnicas evitam o problema do esforço máximo como referência e reduzem, de forma considerável, a variabilidade entre os indivíduos durante a análise do padrão de marcha ${ }^{5}$. O procedimento de referência para uso destes métodos é basicamente em função da amplitude média do sinal da EMG, definida por alguns autores como mean dynamic method ou como peak dynamic method, computados durante o ciclo da marcha ${ }^{4,5,14}$. Conforme o grupo de Winter ${ }^{5}$, as duas técnicas de referência (mean and peak dynamic method) permitem reduzir a variabilidade dos dados, quando comparadas aos métodos de referência por meio da CVM e de contração submáxima. Entretanto, a literatura 
sobre o assunto suporta que essas técnicas empregadas para análise da marcha não preservam necessariamente as verdadeiras variâncias biológicas do grupo muscular avaliado, em razão de estimar a contribuição muscular por meio da média aritmética dos dados ou por meio do pico da atividade muscular durante a própria atividade dinâmica ${ }^{8}$. De fato, para condições dinâmicas, somente o método de normalização por meio da CVM é capaz de preservar as variâncias biológicas e ser sensível o suficiente para detectar as alterações neuromusculares de pacientes durante a marcha ${ }^{8,17,20}$.

Para minimizar os problemas angulares de amplitude articular do movimento durante a marcha, outro método de normalização refere-se ao uso de uma contração isocinética (isokinetic MVC method). Esta técnica foi desenvolvida para avaliar os grupos musculares em ângulos correspondentes aos da tarefa experimental ${ }^{4,12}$. Kellis e Baltzopoulos ${ }^{12}$ demonstraram que o método isokinetic MVC seria o mais eficaz para avaliar a atividade dos músculos do joelho durante tarefas dinâmicas, quando comparado ao estático de CVM. Entretanto, em um estudo mais recente ${ }^{18}$, não se observou nenhuma diferença entre os dois métodos de normalização (CMV isométrica e isokinetic MVC) para análise da marcha e além do que mostrou que o método isokinetic $M V C$ produzia mais variabilidade intra-indivíduo comparativamente aos outros métodos de normalização. A princípio o ângulo arbitrário no modo isométrico não afetaria na estimação e a comparação do padrão de ativação da marcha entre diferentes indivíduos ${ }^{4}$.

Por fim, a predição dos esforços máximos pode ser realizada a partir de medidas antropométricas dos indivíduos. Marras e Davis ${ }^{15}$ mostraram semelhanças entre o verdadeiro valor de torque máximo em relação ao valor predito na equação com base nas características antropométricas de sujeitos saudáveis. Este grupo de pesquisadores sugeriu que este método (torque máximo predito + EMG predito) poderia ser utilizado como referência no procedimento de normalização da EMG. Entretanto, um estudo mais recente mostrou a ineficácia deste em pessoas saudáveis e pacientes com lombalgia crônica ${ }^{27}$. Conforme os resultados da pesquisa, o modelo de regressão linear final utilizado explicava apenas 39\% da variância sobre o produto final (torque máximo) ${ }^{27}$.

Para Mathiassen et al. ${ }^{10}$, a contração de referência, por ela mesma, introduz a variabilidade no sinal da EMG. É provável que a variabilidade entre os indivíduos permaneça, independente de uma técnica de normalização em relação ao outro $^{28}$. É pouco provável que algumas das técnicas utilizadas atualmente na literatura consiga reverter todas as fontes de erros para se conseguir uma medida precisa de atividade muscular. Novos esforços deverão ser colocados em prática com o intuito de se obterem outras formas de normalização da EMG que sejam independente do desempenho individual, mas específica às fontes de variância, tais como o tecido adiposo, distância músculo-eletrodo ${ }^{28,29}$. Nordander et al..$^{28}$ mostraram uma relação negativa entre a EMG e a quantidade de tecido adiposo subcutânea, com o uso da técnica de contração submáxima. Conforme um estudo, $81 \%$ da variância dentro do sinal da EMG poderia ser explicada pela variação do tecido adiposo subcutânea ${ }^{29}$. Entretanto, futuros estudos são necessários para melhor entender os procedimentos adequados a fim de considerar a quantidade de tecido adiposo no procedimento de normalização.

\section{Considerações finais}

Existe um debate na literatura relativo às diferentes técnicas de normalização do sinal da EMG que poderiam ser utilizadas para tarefas estáticas e dinâmicas. Em geral, a escolha de uma técnica de normalização é com base na variabilidade entre e intra-indivíduos. Se o objetivo do estudo for reduzir a variabilidade, então a técnica da contração submáxima é a mais indicada, considerando que esta permite um padrão mais homogêneo da atividade muscular durante uma tarefa específica. Todavia, as desvantagens 
são a perda da verdadeira variância individual e a ausência da informação da atividade muscular necessária para a execução da tarefa em relação à capacidade máxima da pessoa. Somente a técnica de CIVM pode preservar essas informações e melhor discriminar os grupos de indivíduos diferentes, mesmo sendo esses patológicos.

\section{Referênciass}

1. De Luca CJ. The use of surface electromyography in biomechanics. J Appl Biomech. 1997; 13:135-63.

2. Moritani T, Muro M. Motor unit activity and surface electromyogram power spectrum during increasing force of contraction. Eur J Appl Physiol. 1987; 56:260-5.

3. Henneman E, Somjen G, Carpenter DO. Excitability and inhibitibility of motoneurons of different sizes. J Neurophysiol. 196528:599-620.

4. Burden A. How should we normalize electromyograms obtained from healthy participants? What we have learned from over 25 years of research. J Electromyogr Kinesiol. 2010;20:1023-35.

5. Yang JF, Winter DA. Electromyographic amplitude normalization methods: improving their sensitivity as diagnostic tools in gait analysis. Arch Phys Med Rehabil. 1984;65:517-21.

6. Mirka GA. The quantification of EMG normalization error. Ergonomics. 1991;34:343-52.

7. Allison GT, Marshall RN, Singer KP. EMG signal amplitude normalization technique in stretchshortening cycle movements. J Electromyogr Kinesiol. 1993;3:236-44.

8. Knutson LM, Soderberg GL, Ballantyne BT, Clarke WR. A study of various normalization procedures for within day electromyographic data. J Electromyogr Kinesiol. 1994;4:47-59.

9. Attebrant M, Mathiassen SE, Winkel J. Normalizing upper trapezius EMG amplitude: comparison of ramp and constant force procedures. J Elecromyogr Kinesiol. 1995;5(4), 245-50.

10. Mathiassen SE, Winkel J, Hagg GM. Normalization of surface EMG amplitude from the upper trapezius muscle in ergonomic studies - a review. J Electromyogr Kinesiol. 1995;5:197-226.
11. Harms-Ringdahl K, Ekholm J, Schuldt K, Linder J, Ericson MO. Assessment of jet pilots' upper trapezius load calibrated to maximal voluntary contraction and a standardized load. J Electromyogr Kinesiol. 1996;6:67-72.

12. Kellis E, Baltzopoulos V. The effects of normalization method on antagonistic activity patterns during eccentric and concentric isokinetic knee extension and flexion. J Electromyogr Kinesiol. 1996;6:235-45.

13. Zakaria D, Kramer JF, Harburn KL. Reliability of non-normalized and normalized integrated EMG during maximal isometric contractions in females. J Electromyogr Kinesiol. 1996;6:129-35.

14. Burden A, Bartlett R. Normalisation of EMG amplitude: an evaluation and comparison of old and new methods. Med Eng Phys. 1999;21:247-57.

15. Marras WS, Davis KG. A non-MVC normalization technique for the trunk musculature: part 1. Method Development. J Electromyogr Kinesiol. 2001;11:1-9.

16. Marras WS, Davis KG, Maronitis AB. A non-MVC normalization technique for the trunk musculature: Part 2. Validation and use to predict spinal loads. J Electromyogr Kinesiol. 2001;11:11-8.

17. Benoit DL, Lamontagne M, Cerulli G, Liti A. The clinical significance of electromyography normalisation techniques in subjects with anterior cruciate ligament injury during treadmill walking. Gait Posture. 2003;18:56-63.

18. Burden AM, Trew M, Baltzopoulos V. Normalisation of gait EMGs: a re-examination. J Electromyogr Kinesiol. 2003;13:519-32.

19. Ekstrom RA, Soderberg GL, Donatelli RA. Normalization procedures using maximum voluntary isometric contractions for the serratus anterior and trapezius muscles during surface EMG analysis. J Electromyogr Kinesiol. 2005;15:418-28.

20. Milot MH, Nadeau S, Gravel D. Muscular utilization of the plantarflexors, hip flexors and extensors in persons with hemiparesis walking at self-selected and maximal speeds. J Electromyogr Kinesiol. 2007;17:184-93.

21. Oddsson LI, De Luca CJ. Activation imbalances in lumbar spine muscles in the presence of chronic low back pain. J Appl Physiol. 2003;94:1410-20.

22. Arsenault AB, Winter DA, Marteniuk RG. Is there a 'normal' profile of EMG activity in gait? Med Biol Engineer Comput. 1986;24(4):337-43. 
23. Da Silva RA, Lariviere C, Arsenault AB, Nadeau S, Plamondon A. Pelvic stabilization and semisitting position increase the specificity of back exercises. Med Sci Sports Exerc. 2009;41:435-43.

24. Da Silva RA, Lariviere C, Arsenault AB, Nadeau S, Plamondon A. Effect of pelvic stabilization and hip position on trunk extensor activity during back extension exercises on a Roman chair. J Rehabil Med. 2009;41:136-42.

25. Dankaerts W, O'Sullivan PB, Burnett AF, Straker LM, Danneels LA. Reliability of EMG measurements for trunk muscles during maximal and sub-maximal voluntary isometric contractions in healthy controls and CLBP patients. J Electromyogr Kinesiol. 2004;14:333-42.

26. Silfies SP, Squillante D, Maurer P, Westcott S, Karduna AR. Trunk muscle recruitment patterns in specific chronic low back pain populations. Clin Biomech (Bristol, Avon), 2005, 20:465-73.
27. Larivière $\mathrm{C}$, Gravel $\mathrm{D}$, Arsenault $\mathrm{AB}$, Gagnon D, Loisel P, Lepage Y. Back strength cannot be predicted accurately from anthropometric measures in subjects with and without chronic low back pain. Clin Biomech. 2003 Jul;18(6):473-9.

28. Nordander C, Willner J, Hansson GA, Larsson B, Unge J, Granquist L, Skerfving S. Influence of the subcutaneous fat layer, as measured by ultrasound, skinfold calipers and BMI, on the EMG amplitude. Eur J Appl Physiol. 2003;89:514-9.

29. Hemingway MA, Biedermann HJ, Inglis J. Electromyographic recordings of paraspinal muscles: variations related to subcutaneous tissue thickness. Biofeed Self-Regul. 1995;20:39-49. 
